\title{
Effect of statins on cardiovascular events in patients with mild to moderate chronic kidney disease: a systematic review and meta-analysis of randomized clinical trials
}

\author{
Xiao Zhang ${ }^{1+}$, Chun Xiang ${ }^{1+}$, Yu-Hao Zhou ${ }^{2+}$, An Jiang ${ }^{3+}$, Ying-Yi Qin ${ }^{1}$ and Jia He H* $^{*}$
}

\begin{abstract}
Background: Statins are commonly used to lower total cholesterol levels in the general population to prevent cardiovascular events. However, the effects of statins in patients with chronic kidney disease remain unclear. We therefore performed a meta-analysis to assess the effects of statin therapy on cardiovascular outcomes in patients with mild to moderate chronic kidney disease.

Methods: We systematically searched PubMed, EmBase, the Cochrane Central Register of Controlled Trials, proceedings of major meetings, and reference lists of articles for relevant literature. Only randomized clinical trials were included. Outcomes analysed included cardiovascular disease, total mortality, myocardial infarction, stroke, cardiovascular death, and possible drug-related adverse events. Subgroup analyses were also performed based on the population characteristics and clinical indexes.

Results: Twelve trials met our inclusion criteria. Overall, statin therapy resulted in a $24 \%$ reduction in the risk of cardiovascular disease ( $R R=0.76,95 \%$ confidence interval $[\mathrm{Cl}], 0.72-0.80)$, a $21 \%$ reduction in the risk of total mortality ( $\mathrm{RR}=0.79,95 \% \mathrm{Cl}, 0.72-0.86)$, a $34 \%$ reduction in the risk of myocardial infarction $(\mathrm{RR}=0.66,95 \% \mathrm{Cl}$, $0.52-0.83)$, a $30 \%$ reduction in the risk of stroke $(\mathrm{RR}=0.70,95 \% \mathrm{Cl}, 0.57-0.85)$, and a $17 \%$ reduction in the risk of cardiovascular mortality $(R R=0.83,95 \% \mathrm{Cl}, 0.73-0.93)$. No statistically significant drug-related adverse events were noted. Subgroup analysis indicated that some important factors such as baseline creatinine level $\geq 1.5 \mathrm{mg} / \mathrm{dL}$, baseline glomerular filtration rate (GFR), and cardiovascular disease history could affect cardiovascular outcomes.

Conclusion: Statin therapy had a clear effect on cardiovascular disease, total mortality, stroke, and myocardial infarction in patients with mild to moderate renal disease. Subgroup analysis indicated that baseline GFR, baseline creatinine level, and a history of cardiovascular disease might play an important role in the cardiovascular outcomes.
\end{abstract}

Keywords: Statin, Cardiovascular events, Chronic kidney disease, Meta-analysis

\section{Background}

Chronic kidney disease (CKD) is the progressive loss of renal function that occurs over months or even years, and is increasingly recognized as a global public health problem [1-3]. Studies have indicated that patients with CKD have cardiovascular mortality rates at least 10

\footnotetext{
* Correspondence: hejia63@yeah.net

${ }^{\dagger}$ Equal contributors

'Department of Health Statistics, Second Military Medical University, 200433 Shanghai, China

Full list of author information is available at the end of the article
}

times higher than that of the general population [4]. These higher rates increase disease burden and treatment cost. However, little is known about the prevention of cardiovascular disease in patients with CKD. An increasing number of experts suggest statin therapy for the prevention and treatment of cardiovascular disease in patients with CKD [5-7].

Statins can reduce blood cholesterol levels and are confirmed as the first-choice agent in the prevention and treatment of cardiovascular disease in patients with or without a history of cardiovascular disease [8]. Some

\section{Biomed Central}

(c) 2014 Zhang et al.; licensee BioMed Central Ltd. This is an open access article distributed under the terms of the Creative Commons Attribution License (http://creativecommons.org/licenses/by/2.0), which permits unrestricted use, distribution, and reproduction in any medium, provided the original work is properly cited. 
clinicians were concerned about the progression of cardiovascular disease in patients with CKD and extended statin therapy to these patients. They considered CKD to be an important and independent cardiovascular risk factor, and found that patients with mild to moderate renal disease are more likely to develop cardiovascular disease than progress to end-stage kidney disease [9-12]. Laboratory studies proved that the pathophysiology of cardiovascular disease differed from the severity of CKD, and end-stage renal disease occurs too late to improve cardiovascular outcomes [13].

Many clinical trials have investigated the effects and safety of statins in patients with mild to moderate CKD; most of them provided positive results. However, some studies illustrated that statins are useful in treating mild CKD disease but ineffective for moderate CKD when patients were categorized by disease stage [14]. In addition, a meta-analysis reported the beneficial effects of statin therapy in patients with CKD [15], but did not focus on mild to moderate CKD to explore the possible influence of population characteristics, clinical features, and quality of trials. Moreover, more data were added to our studies to provide stronger evidence in consideration of adverse events. Hence, we performed a systematic review and meta-analysis to evaluate the effects of statin therapy on cardiovascular outcomes in patients with predialysis CKD, with the aim to assess the efficacy and safety of statins in patients with mild to moderate CKD.

\section{Methods}

\section{Data sources, search strategy, and selection criteria}

This study was approved by the Ethics Committee of the Second Military Medical University. Randomized, double-blind, controlled trials and trials of statins in the English-language literature were eligible for inclusion in our study. Relevant trials were found with the following procedure:

(1) Electronic searches: We systematically searched PubMed, EmBase, MEDLINE, and the Cochrane Central Register of Controlled Trials for articles published since December 20, 2011. Search terms used were kidney disease, renal disease, nephropathy, nephrosis, 3-hydroxy3-methylglutaryl-coenzyme A, statin, statins, lovastatin, simvastatin, pravastatin, fluvastatin, atorvastatin, rosuvastatin, pitavastatin, cerivastatin, ulinastatin, and pentostatin. (2) Additional resources: We contacted authors by email or telephone to obtain all potentially relevant study details (published or unpublished data) regarding their trials and manually searched for proceedings of international conferences in the Cochrane Cardiovascular Disease Group Specialized Register. In addition, we complemented the search by using the PubMed "related articles" function and the science citation by scrutinizing reference lists of included studies and the cross references of relevant papers. Finally, we also searched for ongoing randomized controlled trials (RCTs) that were registered as completed but not published trials, in the Meta Register of Controlled Trials.

The inclusion criteria for the RCTs were as follows: (1) high-quality RCTs comparing any statin with a placebo, no treatment, or another statin, or RCTs comparing low-dose statins with high-dose statins; (2) RCTs including patients with mild to moderate CKD (stages 1-4) $[16,17]$ including end-stage renal disease (estimated glomerular filtration rate [GFR] $<15 \mathrm{~mL} \cdot \mathrm{min}^{-1} \cdot 1.73 \mathrm{~m}^{-2}$ ), but excluding those with patients on dialysis or those who had a kidney transplant; (3) RCTs with a minimum 8-week follow-up period; and (4) those with a sample size $>50$. The literature search and selection were undertaken independently by two investigators (Xiao-Zhang and Chun-Xiang), and any disagreement between them was mediated by a third investigator (Yu-Hao Zhou) until consensus was reached.

\section{Data extraction and quality assessment}

Data extraction and collection were performed independently by two reviewers (Xiao Zhang and Chun Xiang) using a standardized protocol. Any conflict between the reviewers was resolved by a group discussion, after which the primary authors (Yu-Hao Zhou and Jia He) made the final decision. The data extraction included baseline patient characteristics (age, sex, total cholesterol level, GFR, creatinine level, urinary albumin level, intervention, drug dosage, follow-up duration, and adverse events) and outcomes (cardiovascular events, stroke, myocardial infarction, total mortality, cardiovascular mortality, and possible drug-related adverse events). The quality of the included trials was assessed with the Jadad score [18] on the basis of randomization, concealment of treatment allocation, blinding, completion of follow-up, and use of intention-to-treat analysis.

\section{Statistical analysis}

We allocated the results of each RCT as dichotomous frequency data. We compared the pooled relative risks (RRs) and $95 \%$ confidence intervals [CIs] of cardiovascular disease, total mortality, stroke, myocardial infarction, cardiovascular death, and adverse events between the statin therapy and control groups. Subgroup analyses were performed according to mean age ( $\geq 60$ years or $<60$ years), cardiovascular history (with or without cardiovascular history), diabetes (present or absent), baseline GFR level (60$90 \mathrm{~mL} \cdot \min ^{-1} \cdot 1.73 \mathrm{~m}^{-2}$ or $30-60 \mathrm{~mL} \cdot \min ^{-1} \cdot 1.73 \mathrm{~m}^{-2}$ ), baseline creatinine level ( $\geq 1.5 \mathrm{mg} / \mathrm{dL}$ or $<1.5 \mathrm{mg} / \mathrm{dL})$, baseline total cholesterol level $(\geq 5.0 \mathrm{mmol} / \mathrm{L}$ or $<5.0 \mathrm{mmol} / \mathrm{L})$, baseline low-density lipoprotein (LDL) cholesterol level $(\geq 3.0 \mathrm{mmol} / \mathrm{L}$ or $<3.0 \mathrm{mmol} / \mathrm{L})$, LDL cholesterol lowering 
rate $(\geq 30 \%$ or $<30 \%$ ), and follow-up duration ( $\geq 3$ years or $<3$ years) to investigate the change in the risk estimates with population characteristics and clinical indexes. Both the fixed-effects and random-effects models were used to evaluate the pooled RRs and 95\% CIs for the effects of statin therapy. Although both models yielded similar findings, results from the random-effects model indicated that the true underlying effect varied among the included trials and seemed more conservative $[19,20]$. Heterogeneity of treatment effects between trials was evaluated by the $\mathrm{Q}$ test and I-squared $\left(\mathrm{I}^{2}\right)$ statistic [21]. These indexes assess the percentage of variability across studies that are attributable to heterogeneity rather than chance. Statistical heterogeneity was considered significant when $\mathrm{p}<0.10$ for the chi-square test or $\mathrm{I}^{2}>50 \%$. Several methods were used to check for potential publication bias on the outcomes of cardiovascular disease, total mortality, stroke, myocardial infarction, and cardiovascular mortality. Visual inspection of the funnel plot was performed. The Peters test was used to statistically analyse publication bias [22]. We also conducted a sensitivity analysis by removing each individual study from the meta-analysis. All reported p values were two-sided and $p$ values $<0.05$ were regarded as statistically significant. Statistical analyses were performed using the STATA 11.0 (State Corporation, Lake Way, Texas, USA).

\section{Results}

Trial characteristic

Twelve RCTs were included in our meta-analysis (Figure 1) [14,23-34]. One study was reported in two separate published articles [24,31], and only the recent one was included in our analysis [31]. Four trials [23-26] evaluated the effect of pravastatin, three studies $[14,27,28]$ evaluated the effect of simvastatin, two trials $[29,30]$ evaluated the effect of atorvastatin, one study [31] evaluated the effect of fluvastatin, one study [32] evaluated the effect of lovastatin, and the remaining study [33] evaluated the effect of rosuvastatin. The quality of the trials was assessed using the Jadad score. Overall, two trials had a Jadad score of 5 , one trial had a score of 4 , three trials had a score of 3 , and the remaining six trials had a score of 2. Details on each study are listed in Table 1.

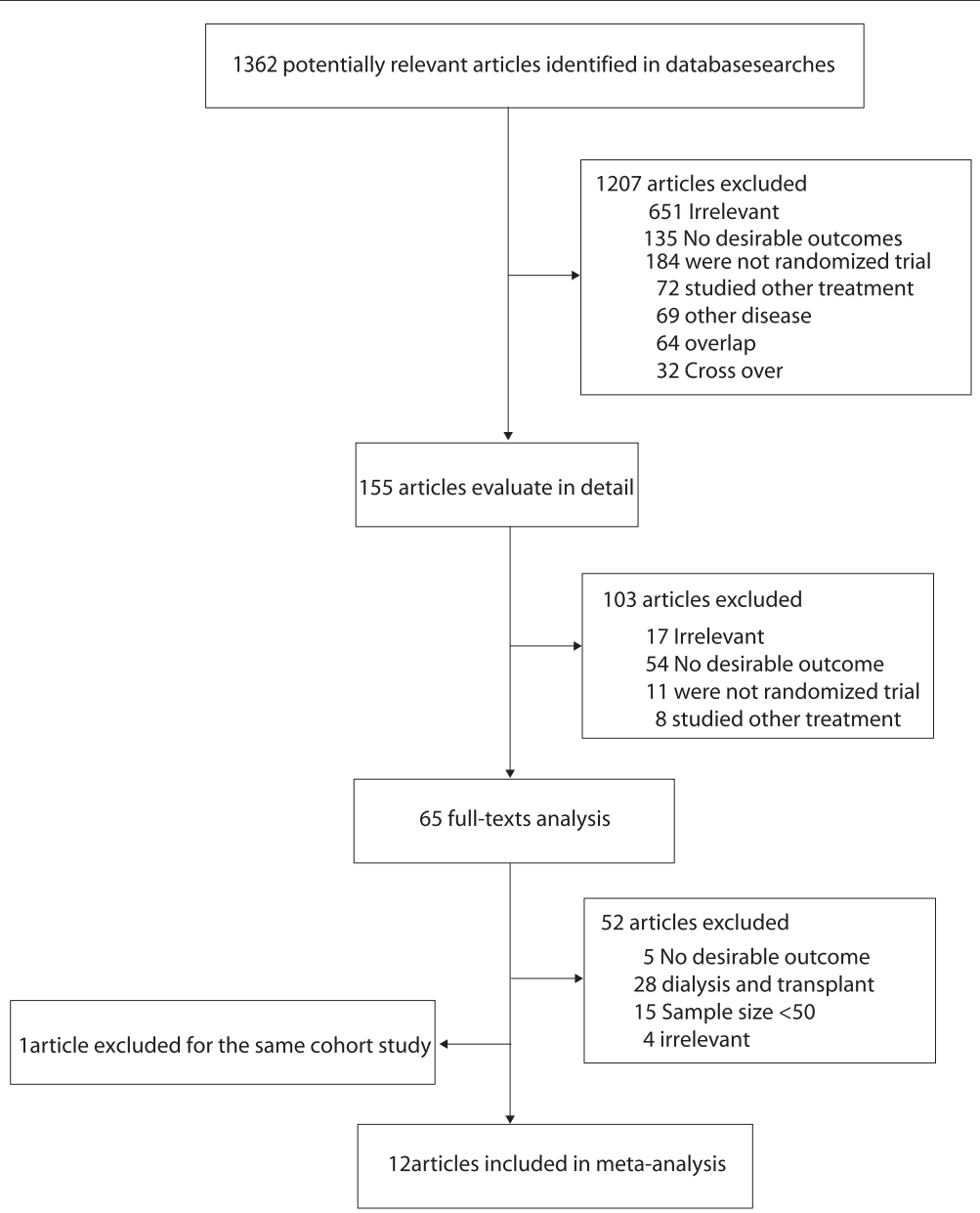

Figure 1 Flow diagram of the literature search and selection process. 
Table 1 Characteristic of studies included in the meta-analysis

\begin{tabular}{|c|c|c|c|c|c|c|c|c|c|c|c|c|c|}
\hline Study & Intervention & Control & $\begin{array}{l}\text { No. of } \\
\text { patient }\end{array}$ & $\begin{array}{l}\text { Gender } \\
\text { (female) }\end{array}$ & $\begin{array}{l}\text { Mean } \\
\text { age, y }\end{array}$ & $\begin{array}{l}\text { Co-morbidity } \\
\text { of CVDs }\end{array}$ & $\begin{array}{l}\text { Co-morbidity of } \\
\text { diabetes }\end{array}$ & $\begin{array}{l}\text { Total cholesterol } \\
(\mathrm{mmol} / \mathrm{L})\end{array}$ & $\begin{array}{c}\text { GFR }(\mathrm{ml} / \mathrm{min} / \\
\left.1.73 \mathrm{~m}^{2}\right)\end{array}$ & $\begin{array}{c}\text { Creatinine } \\
\text { (mg/dL) }\end{array}$ & $\begin{array}{l}\text { LDL } \\
\text { lowing }\end{array}$ & $\begin{array}{l}\text { Follow-up } \\
\text { (month) }\end{array}$ & $\begin{array}{c}\text { Jadad } \\
\text { score }\end{array}$ \\
\hline CARE $(2003)^{34}$ & Pravastatin & Placebo & 1711 & $369(21.6 \%)$ & 64.3 & AMl & NR & 5.40 & $N R$ & 1.26 & $N R$ & 58.9 & 3 \\
\hline $\operatorname{HPS}(2003)^{27 \dagger}$ & Simvastatin & $\begin{array}{l}\text { Vitamins/ } \\
\text { Placebo }\end{array}$ & 1329 & NR & NR & NR & NR & NR & NR & NR & NR & 57.0 & 2 \\
\hline $\operatorname{PPP}(2004)^{23}$ & Pravastatin & Placebo & 16824 & $1840(10.9 \%)$ & 59.5 & $N R$ & NR & 6.05 & 68.5 & 1.18 & $51.1 \%$ & 60.0 & 3 \\
\hline $\begin{array}{l}\text { PREVENDIT } \\
(2004)^{24}\end{array}$ & Pravastatin & $\begin{array}{l}\text { Fosinopril/ } \\
\text { Placebo }\end{array}$ & 864 & $303(35.1 \%)$ & 51.3 & $N R$ & $3 \%$ & 5.80 & $N R$ & 1.02 & $24.4 \%$ & 46.0 & 4 \\
\hline $\operatorname{LIPIS}(2005)^{31}$ & Pravastatin & Placebo & 310 & $102(33 \%)$ & 69.0 & $\mathrm{PCl}$ & NR & 5.17 & $N R$ & 1.33 & $N R$ & 46.0 & 2 \\
\hline $\begin{array}{l}\text { 4S Study } \\
(2007)^{28}\end{array}$ & Simvastatin & Placebo & 2314 & $618(26.7 \%)$ & 60.5 & $\mathrm{CHD}$ & NR & 6.75 & 65.2 & 1.5 & $38.0 \%$ & 65.5 & 2 \\
\hline $\operatorname{ATIC}(2007)^{25}$ & Pravastatin & Placebo & 93 & $40(43.0 \%)$ & 53.0 & $\begin{array}{c}\text { No history of } \\
\text { AOD }\end{array}$ & $\begin{array}{l}\text { Excluded } \\
\text { diabetes }\end{array}$ & 5.60 & 33.5 & 2.32 & $26.1 \%$ & 18.0 & 5 \\
\hline TNT $(2008)^{30}$ & Atorvastatin & Atorvastatin & 3107 & 1005(32.3\%) & 65.5 & $\mathrm{CHD}$ & NR & 4.55 & 52.9 & NR & $18.0 \%$ & 60.0 & 2 \\
\hline $\begin{array}{l}\text { AFCAPS } \\
(2009)\end{array}$ & Lovastatin & Placebo & 304 & $65(21.4 \%)$ & 62.0 & $\begin{array}{c}\text { No history of } \\
\text { CVD }\end{array}$ & $\begin{array}{l}\text { Excluded } \\
\text { diabetes }\end{array}$ & 5.74 & 53.0 & 1.4 & $27.0 \%$ & 64.0 & 3 \\
\hline $\begin{array}{l}\text { ALLIANCE } \\
(2009)^{29}\end{array}$ & Atorvastatin & Usual Care & 579 & 134(23.1\%) & 65.2 & $\mathrm{CHD}$ & NR & 5.89 & 51.2 & 1.5 & $34.5 \%$ & 54.3 & 2 \\
\hline $\operatorname{MEGA}(2009)^{26}$ & Pravastatin & Diet & 7196 & NR & NR & $\begin{array}{l}\text { No history of } \\
\text { CVD }\end{array}$ & NR & NR & 65.0 & NR & $18.5 \%$ & 64.0 & 2 \\
\hline $\begin{array}{l}\text { JUPITER } \\
(2010)^{33}\end{array}$ & Rosuvastatin & Placebo & 3267 & $2129(65.2 \%)$ & 70.0 & NR & $\begin{array}{l}\text { Excluded } \\
\text { diabetes }\end{array}$ & 4.89 & 56.0 & NR & $52.0 \%$ & 22.8 & 2 \\
\hline $\begin{array}{l}\text { SHARP } \\
(2011)^{14}\end{array}$ & Simvastatin & Placebo & 6247 & NR & NR & $\begin{array}{c}\text { No history of } \\
\text { Ml }\end{array}$ & NR & $N R$ & $N R$ & NR & $68.0 \%$ & 58.8 & 5 \\
\hline
\end{tabular}

Abbreviations: $C V D$ cardiovascular disease, $L D L$ low density lipoprotein, $A M I$ acute myocardial infarction, $P C l$ percutaneous coronary intervention, $C H D$ coronary heart disease, $A O D$ arterial occlusive disease, $M I$ myocardial infarction, NR not reported, GFR Glomerular filtration rate. ${ }^{\dagger}$ : only a subgroup of the total study was included. 


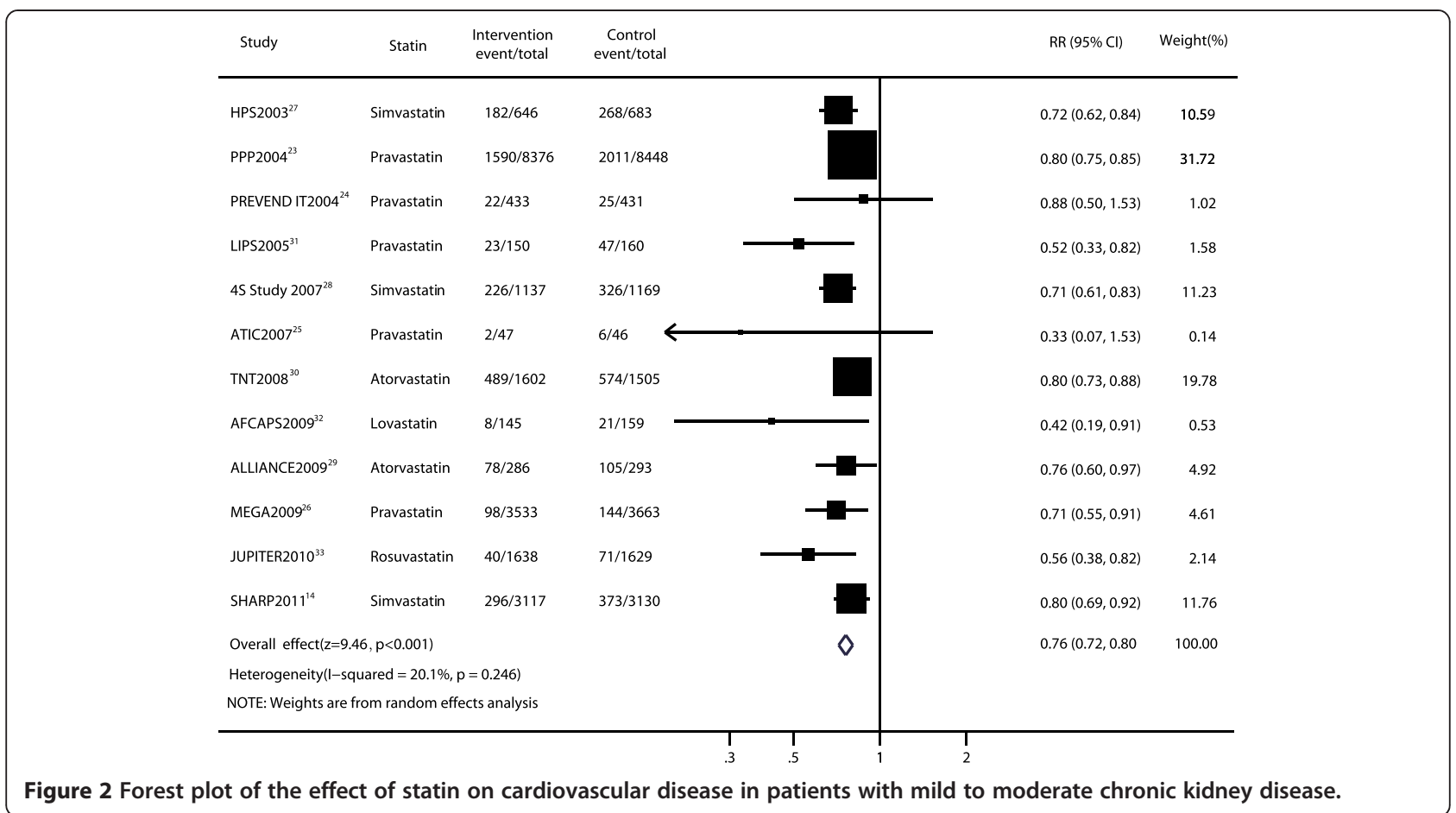

\section{Total effect}

Data for the effect of statin therapy on cardiovascular disease were available from 12 trials [14,23,25-34]. Statin therapy produced a $24 \%$ reduction in the risk of cardiovascular disease (RR, 0.76; 95\% CI, 0.72-0.80; $\mathrm{p}<0.001$; Figure 2) using a random-effects model, and significant heterogeneity was not observed among individual trials $\left(p=0.246 ; I^{2}=20.1 \%\right)$. Sensitivity analyses showed that the RR and 95\% CI were not substantially altered after removal of any single study (data not shown).
The results of the Peters test indicated potential publication bias ( $p=0.005$; Additional file 1: Figure S1a). However, the results of the trim and fill method showed that no trimming was performed and that the pooled result was not changed.

Data for the effect of statin therapy on total mortality were available from seven trials [23,26,28-31,33]. The pooled results revealed that total mortality was significantly lower among patients undergoing stain therapy (RR, 0.79; 95\% CI, 0.72-0.86; Figure 3) by using a

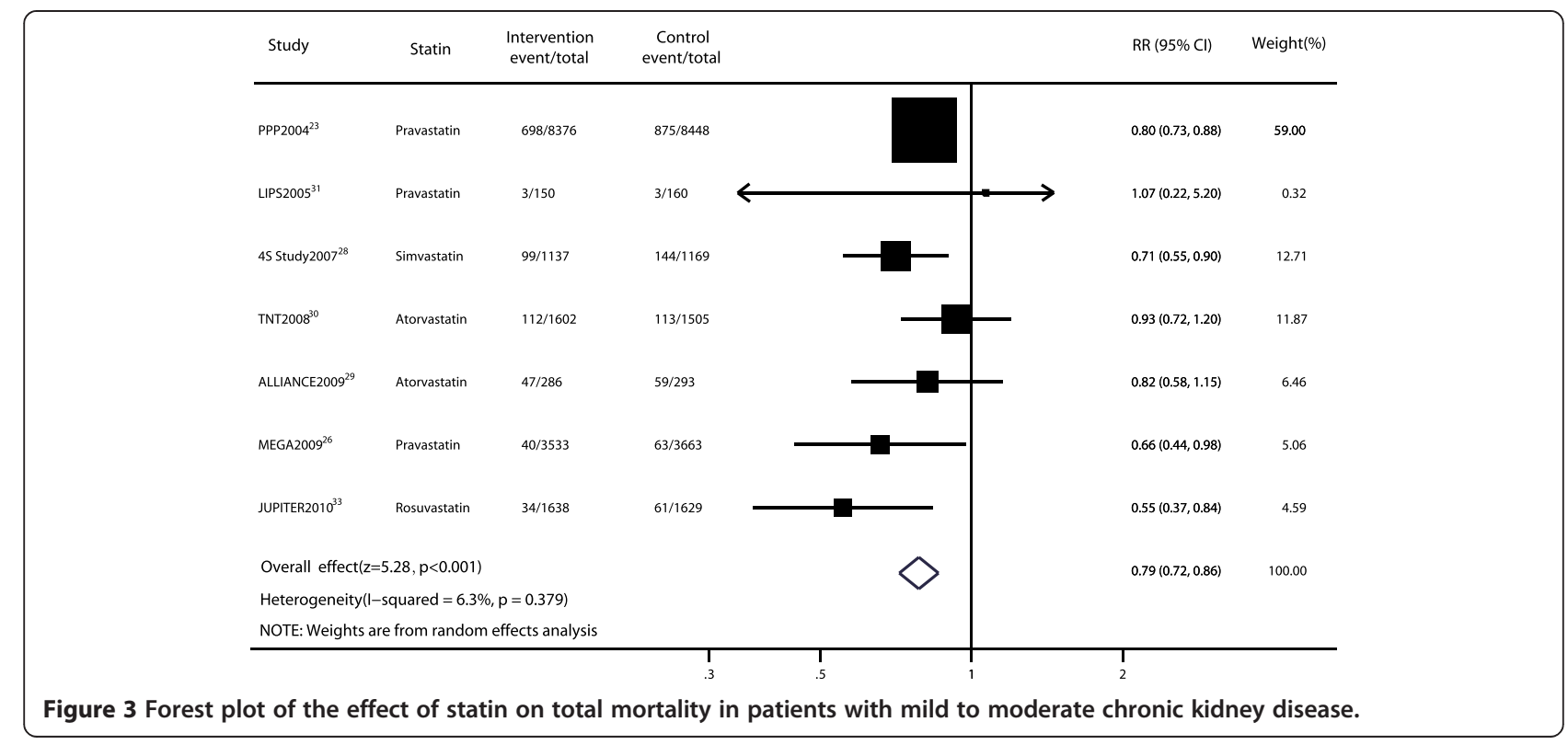




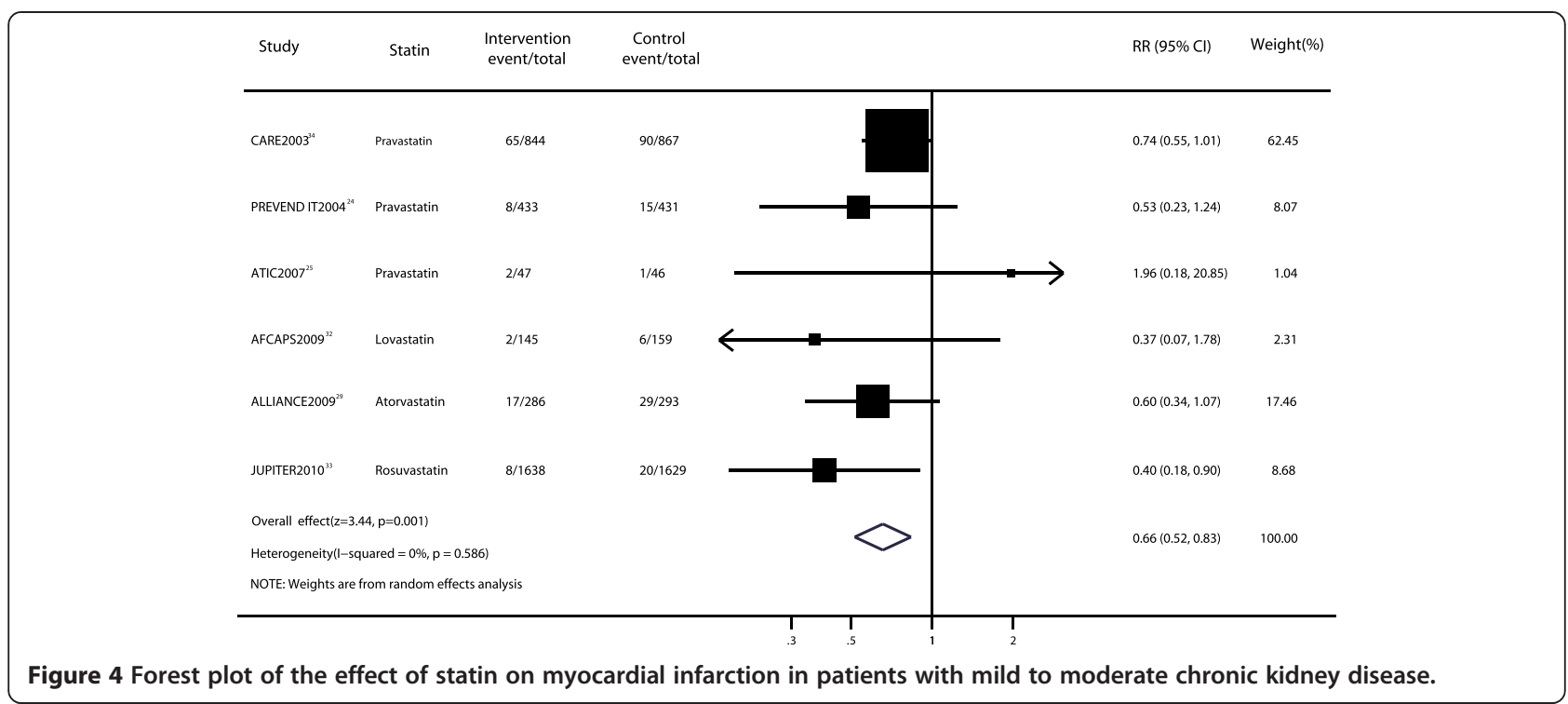

random-effects model. No significant heterogeneity was noted $\left(\mathrm{p}=0.379 ; \mathrm{I}^{2}=6.3 \%\right)$. Sensitivity analyses showed that the RR and 95\% CI were not substantially altered after removal of any single study (data not shown). We found no evidence of publication bias in the pooled analysis of total mortality by using the funnel plot and Peters test ( $\mathrm{p}=0.984$; Additional file 1: Figure S1b).

Six trials provided the data for myocardial infarction [25,29,32-35]. The pooled RR for the effect of myocardial infarction in patients receiving statin therapy compared with that of the control treatment was 0.66 (RR, 0.66; 95\% CI, 0.52-0.83; $\mathrm{p}=0.001$; Figure 4). There was no evidence of significant heterogeneity among individual studies $\left(\mathrm{p}=0.586 ; \mathrm{I}^{2}=0 \%\right)$. Sensitivity analyses showed that the RR and 95\% CI were not substantially altered after removal of any single study (data not shown).
No publication bias was found using the Peters test $(\mathrm{p}=$ 0.618; Additional file 1: Figure S1c).

The effect of statin therapy on stroke was presented in six trials $[26,29,30,33-35]$. The RR was statistically significant in the two groups (RR, 0.70; 95\% CI, 0.57-0.85; $\mathrm{p}<$ 0.001 ; Figure 5) with no significant heterogeneity $(\mathrm{p}=0.715$, $\left.\mathrm{I}^{2}=0 \%\right)$. Sensitivity analyses showed that the RR and 95\% CI were not substantially altered after removal of any single study (data not shown). No evidence of significant publication bias was observed (Peters test, $\mathrm{p}=0.167$; Additional file 1: Figure S1d).

The efficacy of statin therapy for the risk of cardiovascular mortality was presented in six trials [23,25,29,31,32,35]. The results indicated a statistically significant decrease in the risk of cardiovascular death (RR, 0.83; 95\% CI, 0.73$0.93 ; \mathrm{p}=0.002$; Figure 6 ) with no significant heterogeneity

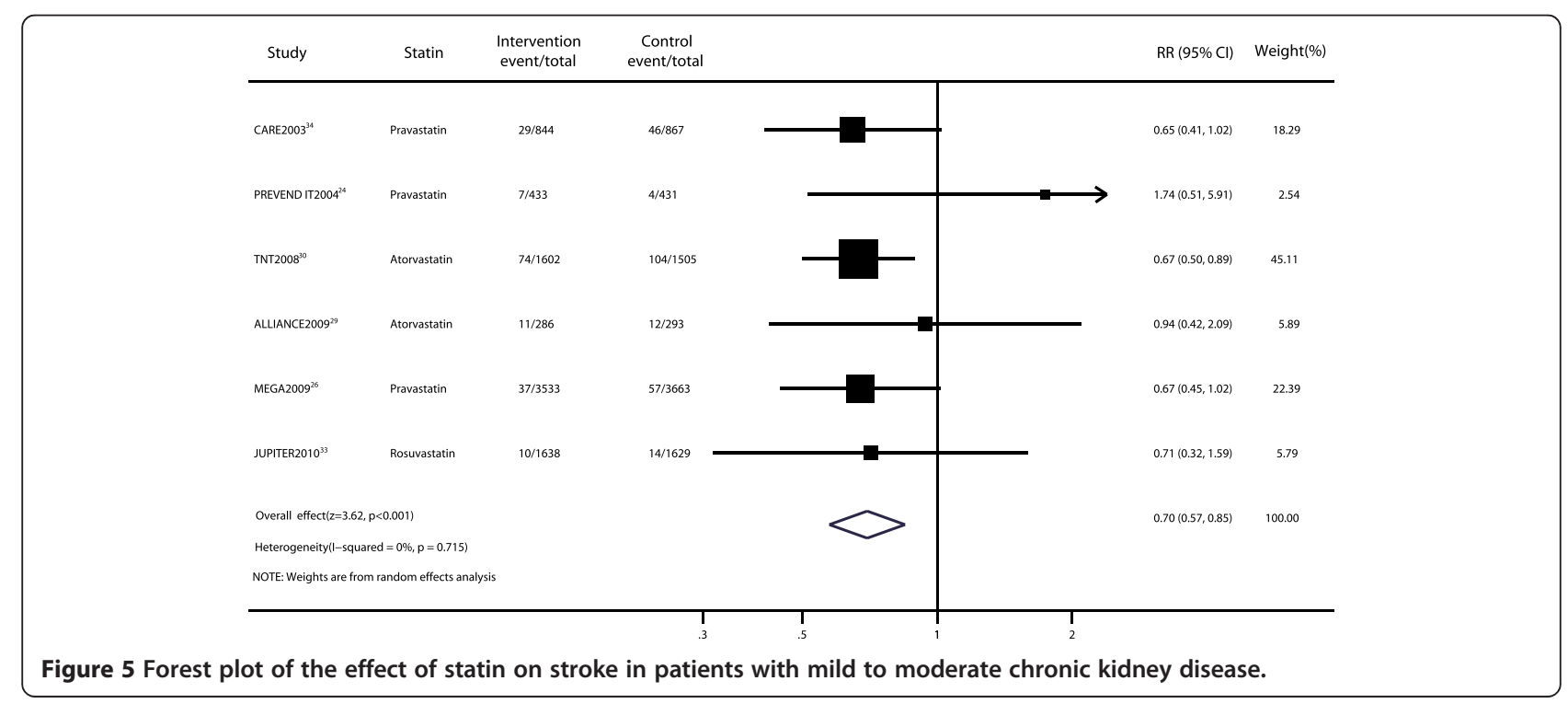




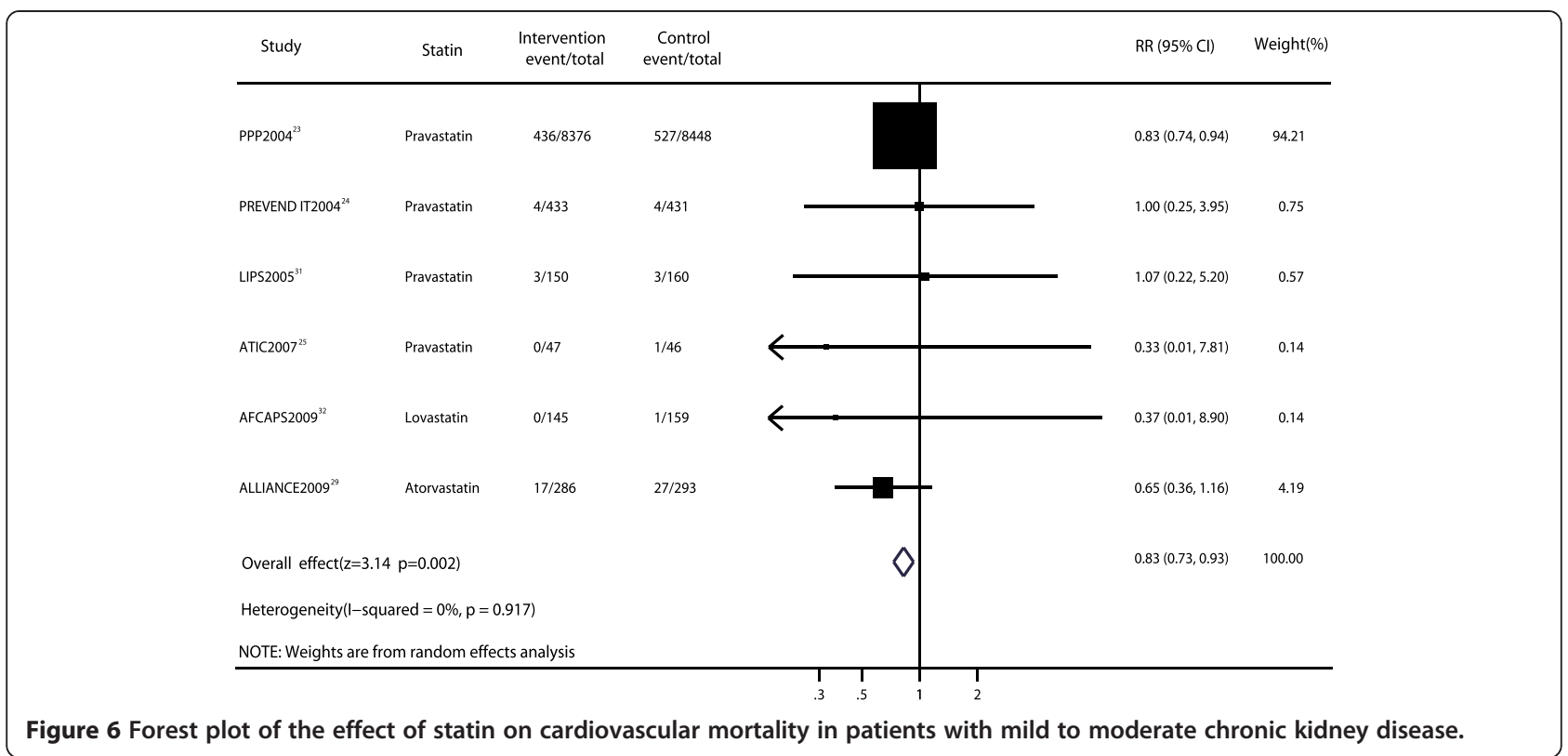

$\left(\mathrm{p}=0.917, \mathrm{I}^{2}=0 \%\right)$. However, after excluding the trial of PPP [23], the outcome was not significant (RR, 0.70; 95\% CI, 0.42-1.14). No evidence of significant publication bias was observed (Peters test, $\mathrm{p}=0.539$; Additional file 1: Figure S1e).

\section{Toxicities}

Five trials reported data on patients who developed elevated serum creatine phosphokinase levels $>3$ or 10 times the upper limit of the normal range [14,26,28,32,34]. No significant differences were seen in the treatment and placebo groups (RR, 1.04; 95\% CI, 0.83-1.31; $\mathrm{p}=0.722$; Figure 7) with no heterogeneity $\left(\mathrm{p}=0.788, \mathrm{I}^{2}=0 \%\right)$.

Two studies provided data on cancer [14,28], and the pooled analysis showed no significant differences for cancer between the statin therapy and control groups (Figure 7). The adverse effect of statin therapy on rhabdomyolysis could be assessed in two trials [28,34], and the pooled

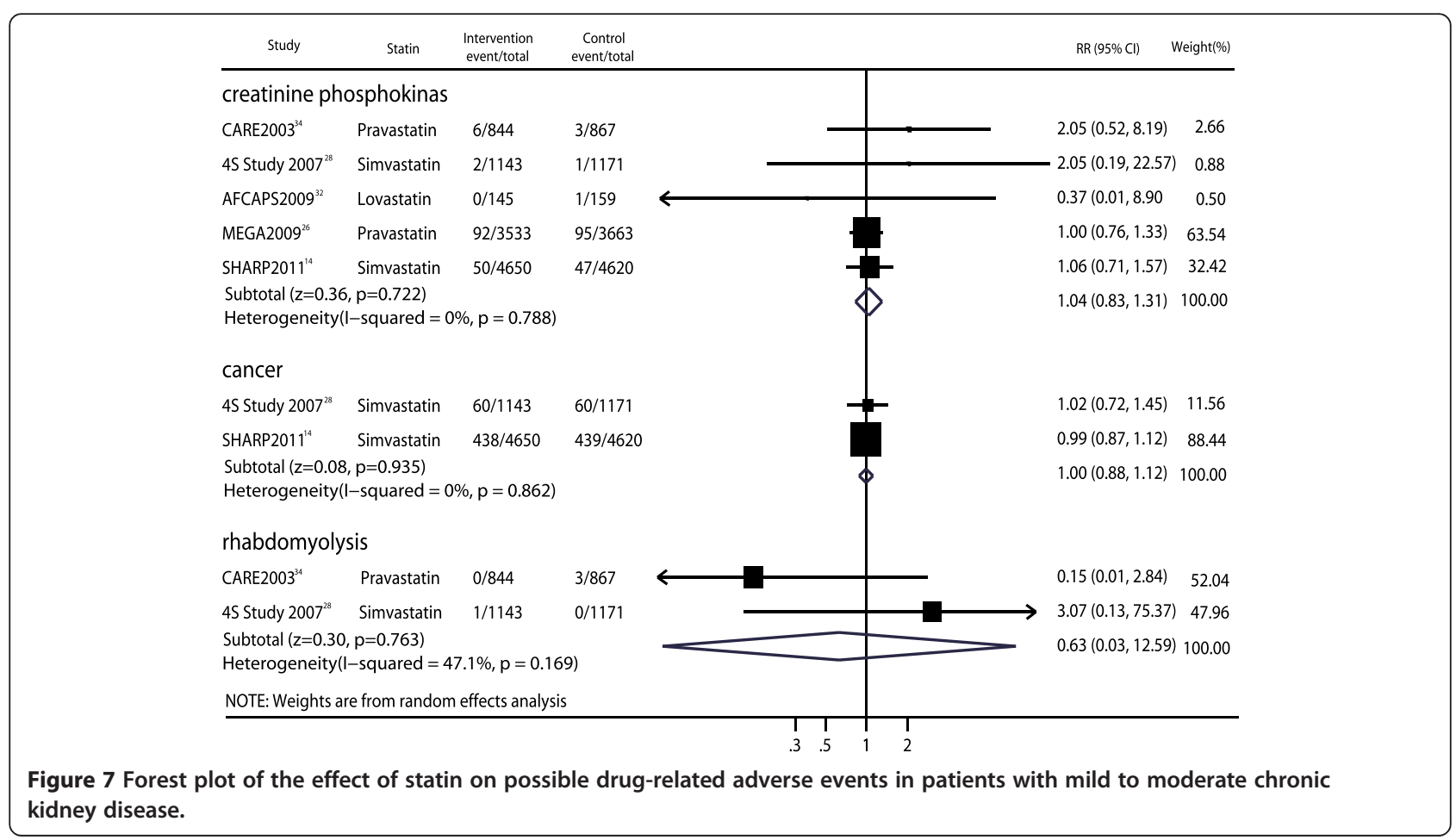


Table 2 Subgroup analysis for the effect of statin therapy on cardiovascular disease

\begin{tabular}{|c|c|c|c|c|c|c|c|c|}
\hline Subgroup & Value & Reference & $\begin{array}{c}\text { Statin } \\
\text { (Event/ } \\
\text { total) }\end{array}$ & $\begin{array}{c}\text { Control } \\
\text { (Event/ } \\
\text { total) }\end{array}$ & $\begin{array}{c}\text { Relative risk } \\
(95 \% \mathrm{Cl})\end{array}$ & $\mathrm{p}$ value & Heterogeneity & $\begin{array}{c}p \text { value for } \\
\text { heterogeneity }\end{array}$ \\
\hline \multirow[t]{2}{*}{ Mean Age } & $>=60$ & $14,27-29,31-33$ & $853 / 7119$ & $1211 / 7223$ & $\begin{array}{c}0.72[0.65 \\
0.79]\end{array}$ & $<0.001$ & $21.2 \%$ & 0.27 \\
\hline & $<60$ & $23-25$ & $1614 / 8856$ & $2042 / 8925$ & $\begin{array}{c}0.80[0.75 \\
0.84]\end{array}$ & $<0.001$ & $0 \%$ & 0.50 \\
\hline \multirow[t]{2}{*}{ Cardiovascular History } & Yes & $28-29,31,34$ & $498 / 2417$ & $712 / 2489$ & $\begin{array}{c}0.72[0.65 \\
0.80]\end{array}$ & $<0.001$ & $0 \%$ & 0.49 \\
\hline & No & $14,25-26,32$ & $404 / 6842$ & $544 / 6998$ & $\begin{array}{c}0.73[0.60 \\
0.88]\end{array}$ & 0.001 & $27.6 \%$ & 0.25 \\
\hline \multirow[t]{2}{*}{ Diabetes } & Yes & $27-28,34$ & $173 / 1334$ & $260 / 1424$ & $\begin{array}{c}0.74[0.63 \\
0.88]\end{array}$ & $<0.001$ & $0 \%$ & 0.94 \\
\hline & No & $25,27-28,32-34$ & $329 / 3123$ & $484 / 3129$ & $\begin{array}{c}0.68[0.59 \\
0.79]\end{array}$ & $<0.001$ & $10 \%$ & 0.35 \\
\hline \multirow[t]{2}{*}{ Baseline GFR } & $>=60$ & $23,26,28$ & 1914/13046 & $2481 / 13280$ & $\begin{array}{c}0.77[0.71 \\
0.83]\end{array}$ & $<0.001$ & $21.9 \%$ & 0.28 \\
\hline & $<60$ & $25,29,32-33$ & $128 / 2116$ & $203 / 2127$ & $\begin{array}{c}0.62[0.47 \\
0.83]\end{array}$ & 0.001 & $29.4 \%$ & 0.24 \\
\hline \multirow[t]{2}{*}{ Baseline Creatinine } & $>=1.5$ & 25,29 & $80 / 333$ & $111 / 339$ & $\begin{array}{l}0.71[0.45 \\
\quad 1.12]\end{array}$ & 0.139 & $11.6 \%$ & 0.29 \\
\hline & $<1.5$ & $23,24,28,31-32$ & 1869/10241 & $2430 / 10367$ & $\begin{array}{c}0.73[0.63 \\
0.84]\end{array}$ & $<0.001$ & $48.0 \%$ & 0.10 \\
\hline \multirow[t]{2}{*}{$\begin{array}{c}\text { Baseline Total } \\
\text { Cholesterol }\end{array}$} & $>=5.0$ & 23,28 & $1816 / 9513$ & 2337/9527 & $\begin{array}{c}0.77[0.70 \\
0.84]\end{array}$ & $<0.001$ & $35.6 \%$ & 0.21 \\
\hline & $<5.0$ & $24-25,29,31-34$ & $344 / 3543$ & $509 / 3585$ & $\begin{array}{c}0.69[0.60 \\
0.80]\end{array}$ & $<0.001$ & $18.1 \%$ & 0.29 \\
\hline \multirow[t]{2}{*}{ Baseline LDL Cholesterol } & $>=3.0$ & $23-25,27-29,31-32$ & $2131 / 11220$ & 2809/11389 & $\begin{array}{c}0.74[0.70 \\
0.81]\end{array}$ & $\begin{array}{l}P< \\
0.001\end{array}$ & $29.0 \%$ & 0.20 \\
\hline & $<3.0$ & $14,30,33$ & $825 / 6357$ & $1018 / 6264$ & $\begin{array}{c}0.76[0.72 \\
0.81]\end{array}$ & $\begin{array}{l}P< \\
0.001\end{array}$ & $37.7 \%$ & 0.20 \\
\hline \multirow[t]{2}{*}{$\begin{array}{l}\text { LDL Cholesterol } \\
\text { Lowering }\end{array}$} & $\begin{array}{l}>= \\
30 \%\end{array}$ & $14,23,26,28,29,33$ & 2328/18087 & $3030 / 18332$ & $\begin{array}{c}0.77[0.73 \\
0.82]\end{array}$ & $\begin{array}{l}P< \\
0.001\end{array}$ & $10.4 \%$ & 0.35 \\
\hline & $<30 \%$ & $24,25,32$ & $32 / 625$ & $52 / 636$ & $\begin{array}{c}0.76[0.71 \\
0.82]\end{array}$ & 0.087 & $35.4 \%$ & 0.21 \\
\hline \multirow[t]{2}{*}{ Follow Up } & $>=3$ & $14,23-24,26-29,31-32$ & $2523 / 17823$ & $3320 / 18136$ & $\begin{array}{c}0.76[0.72 \\
0.81]\end{array}$ & $\begin{array}{l}P< \\
0.001\end{array}$ & $14.4 \%$ & 0.31 \\
\hline & $<3$ & 25,33 & $42 / 1685$ & $77 / 1675$ & $\begin{array}{c}0.74[0.69 \\
0.80]\end{array}$ & 0.001 & $0 \%$ & 0.51 \\
\hline
\end{tabular}

Abbreviations: $C$ confidential interval, GFR glomerular filtration rate, $L D L$ low density lipoprotein.

analysis also showed no significant differences for cancer between patients receiving statin therapy and those receiving the placebo (Figure 7).

\section{Subgroup analysis}

Superior efficacy of statin therapy on cardiovascular events was found in the majority of subgroup analyses, except for patients with a baseline creatinine level $\geq 1.5 \mathrm{mg} / \mathrm{dL}$ (RR, 0.71; 95\% CI, 0.45-1.12; $\mathrm{p}=$ 0.139 ; Table 2). We noted that statin therapy had no effect on total mortality in patients with a history of cardiovascular disease (RR, 1.48; 95\% CI, 0.41-5.36; $\mathrm{p}=0.55)$, baseline GFR $<60 \mathrm{~mL} \cdot \mathrm{min}^{-1} \cdot 1.73 \mathrm{~m}^{-2}(\mathrm{RR}$, 0.68 ; $95 \% \mathrm{CI}, 0.47,1.00 ; \mathrm{p}=0.050$ ), and baseline creatinine level $>1.5 \mathrm{mg} / \mathrm{dL}$ (RR, 0.82; 95\% CI, 0.58-1.15; $\mathrm{p}=$ 0.251 ; Table 3). Additional factors such as age, cardiovascular history, baseline creatinine level, LDL cholesterol lowering rate, and follow-up duration also showed some heterogeneity between groups in the subgroup analysis of myocardial infarction (Table 4). Most of the subgroup analyses on stroke were not effective, except for a follow-up duration $>3$ years (Table 5). None of the subgroup analyses on cardiovascular death was statistically significant (Table 6). 
Table 3 Subgroup analysis for the effect of statin therapy on total mortality

\begin{tabular}{|c|c|c|c|c|c|c|c|c|}
\hline Subgroup & Value & Reference & $\begin{array}{c}\text { Statin } \\
\text { Event/total }\end{array}$ & $\begin{array}{c}\text { Control } \\
\text { Event/total }\end{array}$ & $\begin{array}{c}\text { Relative risk } \\
(95 \% \mathrm{Cl})\end{array}$ & $\begin{array}{c}\mathrm{p} \\
\text { value }\end{array}$ & Heterogeneity & $\begin{array}{c}p \text { value for } \\
\text { heterogeneity }\end{array}$ \\
\hline \multirow[t]{2}{*}{ Mean Age } & $>=60$ & 23 & $183 / 3211$ & $267 / 3251$ & $0.71[0.59,0.84]$ & $<0.001$ & $0 \%$ & 0.52 \\
\hline & $<60$ & $28,29,31,33$ & $698 / 8376$ & $873 / 8448$ & $0.81[0.73,0.89]$ & $<0.001$ & - & - \\
\hline \multirow[t]{2}{*}{ Cardiovascular History } & Yes & $28,29,31,34$ & $235 / 2417$ & $317 / 11689$ & $1.48[0.41,5.36]$ & 0.550 & $98.1 \%$ & $<0.001$ \\
\hline & No & 26 & $40 / 3533$ & $63 / 3663$ & $0.66[0.44,0.98]$ & 0.037 & - & - \\
\hline \multirow[t]{2}{*}{ Baseline GFR } & $>=60$ & $23,26,28$ & $837 / 13046$ & $1082 / 13280$ & $0.78[0.72,0.85]$ & $<0.001$ & $0 \%$ & 0.42 \\
\hline & $<60$ & 29,33 & $47 / 286$ & $59 / 293$ & $0.68[0.47,1.00]$ & 0.050 & $49.8 \%$ & 0.16 \\
\hline \multirow[t]{2}{*}{ Baseline Creatinine } & $>=1.5$ & $23,28,31$ & $47 / 286$ & $59 / 293$ & $0.82[0.58,1.15]$ & 0.251 & - & - \\
\hline & $<1.5$ & 29 & $800 / 9663$ & 1022/9777 & $0.79[0.73,0.87]$ & $<0.001$ & $0 \%$ & 0.58 \\
\hline \multirow[t]{2}{*}{ Baseline Total Cholesterol } & $>=5.0$ & $29,31,33,34$ & $797 / 9513$ & $1017 / 9617$ & $0.75[0.62,0.90]$ & 0.002 & $0 \%$ & 0.45 \\
\hline & $<5.0$ & 23,28 & $282 / 4520$ & $347 / 4454$ & $0.79[0.67,0.93]$ & $<0.001$ & $0 \%$ & 0.33 \\
\hline \multirow[t]{2}{*}{ Baseline LDL Cholesterol } & $>=3.0$ & $23,28-29,31,34$ & $933 / 10793$ & $1192 / 10937$ & $0.79[0.73,0.86]$ & $<0.001$ & $0 \%$ & 0.89 \\
\hline & $<3.0$ & 33 & $34 / 1638$ & $61 / 1629$ & $0.55[0.37,0.84]$ & 0.005 & - & - \\
\hline \multirow[t]{2}{*}{ Follow-up time } & $>=3$ & $23,26,28,29,31,34$ & $973 / 14326$ & $1255 / 14600$ & $0.79[0.73,0.85]$ & $<0.001$ & $0 \%$ & 0.86 \\
\hline & $<3$ & 33 & $34 / 1638$ & $61 / 1629$ & $0.55[0.37,0.84]$ & 0.005 & - & - \\
\hline
\end{tabular}

Abbreviations: $\mathrm{Cl}$ confidential interval, GFR glomerular filtration rate, $L D L$ low density lipoprotein.

\section{Discussion}

Statin is commonly used to lower LDL cholesterol levels in the general population, which reduces the risk of cardiovascular disease $[8,13]$. Moreover, many studies have shown the substantial benefits of statin therapy in patients with cardiovascular disease [36]. However, lack of information on the efficacy and safety of statin therapy in patients with CKD has limited the use of statins for these patients. We excluded patients with end-stage renal disease from our study, which is associated with extremely high cardiovascular morbidity and mortality rates, the co-morbidity of end-stage of renal disease, may be difficult to reverse in this phase $[12,37]$. This study was based on RCTs and investigated any possible correlation between statin therapy and the outcomes of any cardiovascular-related disease in patients with early to moderate renal disease (Kidney Disease Outcomes Quality Initiative [K/DOQI stage 1-4]).

This large quantitative review, including 42,426 individuals with a broad range of baseline characteristics, suggests that statin therapy could lead to a $24 \%$ reduction in the risk of cardiovascular disease, a $21 \%$ reduction in the risk of total mortality, and a $23 \%$ reduction in the risk of cardiovascular mortality in patients with mild to moderate CKD. A previous study indicated that statin therapy reduced the risk of cardiovascular disease, total mortality, and

Table 4 Subgroup analysis for the effect of statin therapy on myocardial infarction

\begin{tabular}{|c|c|c|c|c|c|c|c|c|}
\hline Subgroup & Value & Reference & $\begin{array}{c}\text { Statin } \\
\text { Event/total }\end{array}$ & $\begin{array}{c}\text { Control } \\
\text { Event/total }\end{array}$ & $\begin{array}{l}\text { Relative risk } \\
(95 \% \mathrm{Cl})\end{array}$ & $\begin{array}{c}p \\
\text { value }\end{array}$ & Heterogeneity & $\begin{array}{c}p \text { value for } \\
\text { heterogeneity }\end{array}$ \\
\hline \multirow[t]{2}{*}{ Mean Age } & $>=65$ & $29,32-34$ & $92 / 2913$ & $145 / 2948$ & $0.66[0.51,0.85]$ & 0.001 & $0 \%$ & 0.44 \\
\hline & $<65$ & 24,25 & $10 / 480$ & $16 / 477$ & $0.63[0.27,1.47]$ & 0.283 & $3 \%$ & 0.31 \\
\hline \multirow[t]{2}{*}{ Cardiovascular History } & Yes & 29,34 & $82 / 1130$ & $119 / 1160$ & $0.67[0.14,3.22]$ & 0.012 & $0 \%$ & 0.53 \\
\hline & No & 25,32 & $4 / 192$ & $7 / 205$ & $0.71[0.54,0.93]$ & 0.613 & $25.1 \%$ & 0.25 \\
\hline \multirow[t]{2}{*}{ Baseline Creatinine } & $>=1.5$ & 25,29 & 19/333 & $30 / 339$ & $0.64[0.37,1.12]$ & 0.120 & $0 \%$ & 0.34 \\
\hline & $<1.5$ & $24,32,34$ & $75 / 1422$ & $111 / 1457$ & $0.70[0.53,0.93]$ & 0.013 & $0 \%$ & 0.55 \\
\hline \multirow[t]{2}{*}{ Baseline LDL Cholesterol } & $>=3.0$ & $24,25,29,32,34$ & $94 / 1755$ & $141 / 1796$ & $0.69[0.53,0.88]$ & 0.003 & $0 \%$ & 0.704 \\
\hline & $<3.0$ & 33 & $8 / 1638$ & $20 / 1629$ & $0.40[0.18,0.90]$ & 0.027 & - & - \\
\hline \multirow[t]{2}{*}{ LDL Cholesterol Lowering } & $>=30 \%$ & 29,33 & $25 / 1924$ & 49/1922 & $0.52[0.33,0.84]$ & 0.007 & $0 \%$ & 0.418 \\
\hline & $<30 \%$ & $24,25,32$ & $12 / 625$ & $22 / 636$ & $0.55[0.27,1.13]$ & 0.104 & $0 \%$ & 0.505 \\
\hline \multirow[t]{2}{*}{ Follow-up time } & $>=3$ & $24,29,32,34$ & $92 / 1708$ & $140 / 1750$ & $0.68[0.53,0.87]$ & 0.003 & $0 \%$ & 0.70 \\
\hline & $<3$ & 25,33 & $10 / 1685$ & $21 / 1675$ & $0.59[0.15,2.27]$ & 0.443 & $36 \%$ & 0.21 \\
\hline
\end{tabular}


Table 5 Subgroup analysis for the effect of statin therapy on stoke

\begin{tabular}{|c|c|c|c|c|c|c|c|c|}
\hline Subgroup & Value & Reference & $\begin{array}{c}\text { Statin } \\
\text { (Event/total) }\end{array}$ & $\begin{array}{c}\text { Control } \\
\text { (Event/total) }\end{array}$ & $\begin{array}{c}\text { Relative risk } \\
(95 \% \mathrm{Cl})\end{array}$ & $\begin{array}{c}p \\
\text { value }\end{array}$ & Heterogeneity & $\begin{array}{c}p \text { value for } \\
\text { heterogeneity }\end{array}$ \\
\hline \multirow[t]{2}{*}{ Mean Age } & $>=60$ & $29,33,34$ & $50 / 2768$ & $72 / 2789$ & $0.71[0.50,1.01]$ & 0.058 & $0 \%$ & 0.732 \\
\hline & $<60$ & 24 & $7 / 433$ & $4 / 431$ & $1.74[0.51,5.91]$ & 0.373 & - & - \\
\hline \multirow[t]{2}{*}{ Cardiovascular History } & Yes & 29,34 & $40 / 1130$ & $58 / 1160$ & $0.71[0.48,1.05]$ & 0.088 & $0 \%$ & 0.429 \\
\hline & No & 26 & $37 / 3533$ & $57 / 3663$ & $0.67[0.45,1.02]$ & 0.059 & - & - \\
\hline \multirow[t]{2}{*}{ Baseline GFR } & $>=60$ & 26 & $37 / 3533$ & $57 / 3663$ & $0.67[0.45,1.02]$ & 0.059 & - & - \\
\hline & $<60$ & 29,33 & $21 / 1924$ & $26 / 1922$ & $0.82[0.46,1.45]$ & 0.488 & $0 \%$ & 0.63 \\
\hline \multirow[t]{2}{*}{ Baseline Creatinine } & $>=1.5$ & 29 & $11 / 286$ & $12 / 293$ & $0.94[0.42,2.09]$ & 0.878 & & \\
\hline & $<1.5$ & 24,34 & $36 / 1277$ & $50 / 1298$ & $0.90[0.36,2.23]$ & 0.815 & $54.9 \%$ & 0.14 \\
\hline \multirow[t]{2}{*}{ Baseline LDL Cholesterol } & $>=3.0$ & $24,29,34$ & $47 / 1563$ & $62 / 1591$ & $0.82[0.51,1.30]$ & 0.387 & $20.3 \%$ & 0.29 \\
\hline & $<3.0$ & 33 & 10/1638 & $14 / 1429$ & $0.71[0.32,1.60]$ & 0.407 & - & - \\
\hline \multirow[t]{2}{*}{ LDL Cholesterol Lowering } & $>=30 \%$ & 29,33 & 21/1924 & 26/1922 & $0.82[0.46,1.45]$ & 0.488 & $0 \%$ & 0.63 \\
\hline & $<30 \%$ & 24,26 & $44 / 3966$ & $61 / 4094$ & $0.90[0.38,2.14]$ & 0.818 & $52.2 \%$ & 0.15 \\
\hline \multirow[t]{2}{*}{ Follow-up time } & $>=3$ & $24,26,29,34$ & $84 / 5096$ & $119 / 5254$ & $0.73[0.55,0.96]$ & 0.023 & $0 \%$ & 0.43 \\
\hline & $<3$ & 33 & $10 / 1638$ & $14 / 1629$ & $0.71[0.32,1.59]$ & 0.407 & - & - \\
\hline
\end{tabular}

Abbreviations: $\mathrm{Cl}$ confidential interval, GFR glomerular filtration rate, $L D L$ low density lipoprotein.

cardiovascular death in a subgroup analysis of pre-dialysis patients [15]. These findings were similar to the results of our study. Our main findings also support the conclusion of the Pravastatin Pooling Project (PPP 2004), which was a pooled analysis of three major statin studies on the subject level and demonstrated that statin therapy also appeared to reduce the incidence of cardiovascular disease and total mortality rate in patients with mild to moderate CKD [23]. Furthermore, our study also reported the results of myocardial infarction and stroke and concluded that statin therapy also contributed to a $34 \%$ reduction in the risk of myocardial infarction and a 30\% reduction in the risk of stroke.

The outcome of cardiovascular disease, cardiovascular death, and total mortality was also reported in two previous meta-analyses [15,38]. Our meta-analysis extended this by including 8 additional randomized trials with patients across a wider background of cardiovascular risk $[14,25,26,28-30,32,33]$. We also performed more detailed subgroup analysis to explore whether the effects varied among different patients. We did not include trials with small sample size or short duration because cardiovascular events and CKD might not be clearly defined in these small trials, or the risk for outcome might not be accurate owing to insufficient observation times.

Some important factors such as baseline creatinine level, baseline GFR, and cardiovascular disease history may affect the results. The effects of statin therapy on cardiovascular disease in patients with baseline creatinine levels $>1.5 \mathrm{mg} /$ dL was not statistically significant. Similar effects were also

Table 6 Subgroup analysis for the effect of statin therapy on cardiovascular death

\begin{tabular}{|c|c|c|c|c|c|c|c|c|}
\hline Subgroup & Value & Reference & $\begin{array}{c}\text { Statin } \\
\text { (Event/total) }\end{array}$ & $\begin{array}{c}\text { Control } \\
\text { (Event/total) }\end{array}$ & $\begin{array}{c}\text { Relative risk } \\
(95 \% \mathrm{Cl})\end{array}$ & $\begin{array}{c}p \\
\text { value }\end{array}$ & Heterogeneity & $\begin{array}{c}p \text { value for } \\
\text { heterogeneity }\end{array}$ \\
\hline \multirow[t]{2}{*}{ Mean Age } & $>=65$ & 24,25 & $76 / 1425$ & 99/1479 & $0.79[0.59,1.06]$ & 0.114 & $0 \%$ & 0.81 \\
\hline & $<65$ & $29,31,32,34$ & $4 / 480$ & $5 / 477$ & $0.83[0.24,2.96]$ & 0.778 & $0 \%$ & 0.53 \\
\hline \multirow[t]{2}{*}{ Cardiovascular History } & Yes & $29,31,34$ & $76 / 1280$ & $98 / 1230$ & $0.80[0.60,1.07]$ & 0.126 & $0 \%$ & 0.69 \\
\hline & No & 25,32 & 0/192 & $2 / 205$ & $0.35[0.04,3.28]$ & 0.354 & $0 \%$ & 0.96 \\
\hline \multirow[t]{2}{*}{ Baseline Creatinine } & $>=1.5$ & 25,29 & $17 / 333$ & 28/339 & $0.63[0.35,1.12]$ & 0.116 & $0 \%$ & 0.68 \\
\hline & $<1.5$ & $24,31,32,34$ & $63 / 1572$ & 76/1617 & $0.85[0.62,1.18]$ & 0.338 & $0 \%$ & 0.94 \\
\hline \multirow[t]{2}{*}{ LDL Cholesterol Lowering } & $>=30 \%$ & 29 & $17 / 286$ & $27 / 293$ & $0.65[0.36,1.16]$ & 0.142 & - & - \\
\hline & $<30 \%$ & $24,25,32$ & $4 / 625$ & $6 / 636$ & $0.75[0.23,2.42]$ & 0.625 & $0 \%$ & 0.73 \\
\hline \multirow[t]{2}{*}{ Follow-up time } & $>=3$ & $24,29,31,32,34$ & $80 / 1858$ & 103/1910 & $0.80[0.60,1.06]$ & 0.122 & $0 \%$ & 0.90 \\
\hline & $<3$ & 25 & $0 / 47$ & $1 / 46$ & $0.33[0.01,7.81]$ & 0.489 & - & - \\
\hline
\end{tabular}


found in a subgroup analysis of total mortality in patients with baseline creatinine levels $>1.5 \mathrm{mg} / \mathrm{dL}$, baseline GFR $<60 \mathrm{~mL} \cdot \mathrm{min}^{-1} \cdot 1.73 \mathrm{~m}^{-2}$, and a cardiovascular disease history. The results emphasized that statin therapy should be examined more carefully in these particular cases. However, further studies are needed to substantiate these conclusions.

A subgroup analysis of baseline LDL cholesterol levels and LDL lowering rates in patients with cardiovascular disease indicated no significant differences, although there were some benefits in the prevention of cardiovascular risk in total analysis. A possible reason could be that although LDL cholesterol level is considered an important risk factor for cardiovascular disease in patients, this strong correlation was attenuated by non-traditional cardiovascular risk factors such as the severity of kidney disease, oxidative stress, and inflammation in patients with renal insufficiency $[32,33,36,39,40]$. This may suggest that cardiovascular risk in patients with CKD, which is a complex mechanism, does not have a simple connection with dyslipidaemia, and may be a less effective indicator of dyslipidaemia as the major causal factor. Therefore, the benefits of statin therapy on cardiovascular disease in patients with mild to moderate CKD could not be explained by a differential action on lipid levels.

The effect of statin therapy on renal function could be qualitatively evaluated in nine included studies [24-26,28-33]. Five studies [14,24,31-33] have reported stable or no adverse effects on kidney function. Moreover, three trials $[25,26,29]$ reported the renoprotective effect observed in the treatment group compared with the placebo group, and only one trial [27] reported adverse effects on kidney function. A possible reason could be that statin therapy reduces inflammation, which may slow the progression of tubulointerstitial nephritis, although a transient increase in proteinuria may be caused, and may protect against long-term renal damage [41]. Therefore, we may conclude that statin therapy has no serious adverse effects on kidney function, and is possibly beneficial to kidney function; however, these arguments definitely need further study with more detailed data on kidney function at the patient level.

Our analysis also found that statin therapy has no significant drug-related side effects such as rhabdomyolysis, cancer, or change in creatine phosphokinase levels compared with the control group. However, these conclusions might be unreliable because only a small number of trials were included in such subsets.

Our study also has some potential limitations. First, our result was based on published data, whereas individual patient data and original data were not available, which may limit the effective assessment of drug effects and safety. Second, the evaluation index of renal function differed in the included trials, which may affect the classification of CKD. Third, baseline characteristics such as the statin type, dosage, disease history, severity of CKD, and follow-up duration varied among included trials, which may have caused some heterogeneity. Last, publication bias was unavoidable in our study.

\section{Conclusion}

In conclusion, our findings strongly suggest that statin therapy has significant benefits in preventing cardiovascular disease, stroke, myocardial infarction, and all-cause mortality in patients with mild to moderate CKD. Furthermore, our study also provides detailed evidence on the prevention of cardiovascular events in patients with different baseline characteristics and renal kidney function, which is needed for physicians to consider the results more carefully and comprehensively in clinical practice. We suggest that the ongoing trials be improved in the following ways: (i) promising interventions should be tested, including dosage, treatment duration, or a combination of these with influencing factors; (ii) details of adverse events of trials should be reported and recorded to allow evaluation of the side effects of any treatment in future trials.

\section{Additional file}

Additional file 1: Figure S1. Funnel plot of log relative risk vs. standard error of log relative risks. (a) Funnel plot for cardiovascular disease; (b) Funnel plot for total mortality; (c) Funnel plot for stoke; (d) Funnel plot for myocardial infarction; (e) Funnel plot for cardiovascular mortality.

\section{Competing interests}

The authors declare that they have no competing interests.

\section{Authors' contributions}

Conception/Design: JH, XZ, Y-HZ. Collection and/or assembly of data: XZ, CX, Y-YQ. Data analysis and interpretation: $X Z, Y-H Z, C X, A J$. Manuscript writing: $X Z$, $Y-H Z$. Final approval of the version to be published: $J H, X Z, C X, Y-H Z, A J, Y-Y Q$.

\section{Funding}

This work is supported by the grants from three Ministry of science and technology of China (2008ZX10002-007,2008ZX10002-018 and 2008ZX10002025), the leading talents of science in Shanghai 2010(022), the key discipline construction of evidence-based public health in Shanghai (12GWZX0602) and the National Natural Science Foundation of China (81373105). The funders had no role in the study design, data collection, analysis, decision to publish, or preparation of the manuscript. The corresponding author had full access to all the data in the study and had final responsibility for the decision to submit for publication.

\section{Author details}

${ }^{1}$ Department of Health Statistics, Second Military Medical University, 200433 Shanghai, China. ${ }^{2}$ Department of Rehabilitation Institute, Shanghai Seventh People's Hospital, Shanghai, China. ${ }^{3}$ Office of Educational Administration, Second Military Medical University, Shanghai, China.

Received: 28 September 2012 Accepted: 11 February 2014 Published: 17 February 2014

\section{Reference}

1. Levey AS, Atkins R, Coresh J, Cohen EP, Collins AJ, Eckardt KU, Nahas ME, Jaber BL, Jadoul M, Levin A, Powe NR, Rossert J, Wheeler DC, Lameire N, Eknoyan G: Chronic kidney disease as a global public health problem: approaches and initiatives - a position statement from kidney disease improving global outcomes. Kidney Int 2007, 72(3):247-259. 
2. Coresh J, Selvin E, Stevens LA, Manzi J, Kusek JW, Eggers P, Van Lente F, Levey AS: Prevalence of chronic kidney disease in the United States. JAMA 2007, 298(17):2038-2047

3. Zhang QL, Rothenbacher D: Prevalence of chronic kidney disease in population-based studies: systematic review. BMC Public Health 2008, 8:117.

4. Gansevoort RT, Correa-Rotter R, Hemmelgarn BR, Jafar TH, Heerspink HJ, Mann JF, Matsushita K, Wen CP: Chronic kidney disease and cardiovascular risk: epidemiology, mechanisms, and prevention. Lancet 2013, 382(9889):339-352.

5. Barsoum RS: Chronic kidney disease in the developing world. N Engl J Med 2006, 354(10):997-999.

6. Chen J, Wildman RP, Gu D, Kusek JW, Spruill M, Reynolds K, Liu D, Hamm $\mathrm{LL}$, Whelton PK, He J: Prevalence of decreased kidney function in Chinese adults aged 35 to 74 years. Kidney Int 2005, 68(6):2837-2845

7. Zhang L, Zhang P, Wang F, Zuo L, Zhou Y, Shi Y, Li G, Jiao S, Liu Z, Liang W, Wang $\mathrm{H}$ : Prevalence and factors associated with CKD: a population study from Beijing. Am J Kidney Dis 2008, 51(3):373-384.

8. Baigent C, Blackwell L, Emberson J, Holland LE, Reith C, Bhala N, Peto R, Barnes EH, Keech A, Simes J, Collins R: Efficacy and safety of more intensive lowering of LDL cholesterol: a meta-analysis of data from 170,000 participants in 26 randomised trials. Lancet 2010, 376(9753):1670-1681.

9. Sarnak MJ, Levey AS, Schoolwerth AC, Coresh J, Culleton B, Hamm LL, McCullough PA, Kasiske BL, Kelepouris E, Klag MJ, Parfrey P, Pfeffer M, Raij L, Spinosa DJ, Wilson PW: Kidney disease as a risk factor for development of cardiovascular disease: a statement from the american heart association councils on kidney in cardiovascular disease, high blood pressure research, clinical cardiology, and epidemiology and prevention. Circulation 2003, 108(17):2154-2169.

10. Weiner DE, Tighiouart H, Amin MG, Stark PC, MacLeod B, Griffith JL, Salem DN, Levey AS, Sarnak MJ: Chronic kidney disease as a risk factor for cardiovascular disease and all-cause mortality: a pooled analysis of community-based studies. J Am Soc Nephrol 2004, 15(5):1307-1315.

11. Culleton BF, Larson MG, Wilson PW, Evans JC, Parfrey PS, Levy D: Cardiovascular disease and mortality in a community-based cohort with mild renal insufficiency. Kidney Int 1999, 56(6):2214-2219.

12. Baigent $C$, Burbury $K$, Wheeler D: Premature cardiovascular disease in chronic renal failure. Lancet 2000, 356(9224):147-152.

13. Szummer K, Lundman P, Jacobson SH, Schon S, Lindback J, Stenestrand U, Wallentin $L$, Jernberg T: Association between statin treatment and outcome in relation to renal function in survivors of myocardial infarction. Kidney Int 2011, 79(9):997-1004

14. Baigent C, Landray MJ, Reith C, Emberson J, Wheeler DC, Tomson C, Wanner C, Krane V, Cass A, Craig J, Neal B, Jiang L, Hooi LS, Levin A, Agodoa L, Gaziano M, Kasiske B, Walker R, Massy Z A, Feldt-Rasmussen B, Krairittichai U, Ophascharoensuk V, Fellstrom B, Holdaas H, Tesar V, Wiecek A, Grobbee D, de Zeeuw D, Gronhagen-Riska C, Dasgupta T, et al: The effects of lowering LDL cholesterol with simvastatin plus ezetimibe in patients with chronic kidney disease (study of heart and renal protection): a randomised placebo-controlled trial. Lancet 2011, 377(9784):2181-2192.

15. Strippoli GF, Navaneethan SD, Johnson DW, Perkovic V, Pellegrini F, Nicolucci A, Craig JC: Effects of statins in patients with chronic kidney disease: meta-analysis and meta-regression of randomised controlled trials. BMJ 2008, 336(7645):645-651.

16. Levey AS, Eckardt KU, Tsukamoto Y, Levin A, Coresh J, Rossert J, De Zeeuw D, Hostetter TH, Lameire N, Eknoyan G: Definition and classification of chronic kidney disease: a position statement from kidney disease: improving global outcomes (KDIGO). Kidney Int 2005, 67(6):2089-2100.

17. National Kidney Foundation: K/DOQI clinical practice guidelines for chronic kidney disease: evaluation, classification, and stratification. Am J Kidney Dis 2002, 39(2 Suppl 1):S1-266.

18. Jadad AR, Moore RA, Carroll D, Jenkinson C, Reynolds DJ, Gavaghan DJ, McQuay HJ: Assessing the quality of reports of randomized clinical trials: is blinding necessary? Control Clin Trials 1996, 17(1):1-12.

19. Ades $A E$, Lu G, Higgins JP: The interpretation of random-effects metaanalysis in decision models. Med Decis Making 2005, 25(6):646-654.

20. DerSimonian R, Laird N: Meta-analysis in clinical trials. Control Clin Trials 1986, 7(3):177-188

21. Higgins JP, Thompson SG, Deeks JJ, Altman DG: Measuring inconsistency in meta-analyses. BMJ 2003, 327(7414):557-560.

22. Peters JL, Sutton AJ, Jones DR, Abrams KR, Rushton L: Comparison of two methods to detect publication bias in meta-analysis. JAMA 2006, 295(6):676-680.

23. Tonelli M, Isles C, Curhan GC, Tonkin A, Pfeffer MA, Shepherd J, Sacks FM, Furberg C, Cobbe SM, Simes J, Craven T, West M: Effect of pravastatin on cardiovascular events in people with chronic kidney disease. Circulation 2004, 110(12):1557-1563.

24. Asselbergs FW, Diercks GF, Hillege HL, van Boven AJ, Janssen WM, Voors AA, de Zeeuw D, de Jong PE, van Veldhuisen DJ, van Gilst WH: Effects of fosinopril and pravastatin on cardiovascular events in subjects with microalbuminuria. Circulation 2004, 110(18):2809-2816.

25. Nanayakkara PW, van Guldener C, ter Wee PM, Scheffer PG, van Ittersum FJ, Twisk JW, Teerlink T, van Dorp W, Stehouwer CD: Effect of a treatment strategy consisting of pravastatin, vitamin E, and homocysteine lowering on carotid intima-media thickness, endothelial function, and renal function in patients with mild to moderate chronic kidney disease: results from the anti-oxidant therapy in chronic renal insufficiency (ATIC) study. Arch Intern Med 2007, 167(12):1262-1270.

26. Nakamura H, Mizuno K, Ohashi Y, Yoshida T, Hirao K, Uchida Y: Pravastatin and cardiovascular risk in moderate chronic kidney disease. Atherosclerosis 2009, 206(2):512-517.

27. Collins R, Armitage J, Parish S, Sleigh P, Peto R: MRC/BHF heart protection study of cholesterol-lowering with simvastatin in 5963 people with diabetes: a randomised placebo-controlled trial. Lancet 2003, 361(9374):2005-2016.

28. Chonchol M, Cook T, Kjekshus J, Pedersen TR, Lindenfeld J: Simvastatin for secondary prevention of all-cause mortality and major coronary events in patients with mild chronic renal insufficiency. Am J Kidney Dis 2007, 49(3):373-382.

29. Koren MJ, Davidson MH, Wilson DJ, Fayyad RS, Zuckerman A, Reed DP: Focused atorvastatin therapy in managed-care patients with coronary heart disease and CKD. Am J Kidney Dis 2009, 53(5):741-750.

30. Shepherd J, Kastelein JJ, Bittner V, Deedwania P, Breazna A, Dobson S, Wilson DJ, Zuckerman A, Wenger NK: Intensive lipid lowering with atorvastatin in patients with coronary heart disease and chronic kidney disease: the TNT (treating to New targets) study. J Am Coll Cardiol 2008, 51(15):1448-1454.

31. Lemos PA, Serruys PW, de Feyter P, Mercado NF, Goedhart D, Saia F, Arampatzis CA Soares PR, Ciccone M, Arquati M, Cortellaro M, Rutsch W, Legrand V: Long-term fluvastatin reduces the hazardous effect of renal impairment on four-year atherosclerotic outcomes (a LIPS substudy). Am J Cardiol 2005, 95(4):445-451.

32. Kendrick J, Shlipak MG, Targher G, Cook T, Lindenfeld J, Chonchol M: Effect of lovastatin on primary prevention of cardiovascular events in mild CKD and kidney function loss: a post hoc analysis of the Air force/texas coronary atherosclerosis prevention study. Am J Kidney Dis 2010, 55(1):42-49.

33. Ridker PM, MacFadyen J, Cressman M, Glynn RJ: Efficacy of rosuvastatin among men and women with moderate chronic kidney disease and elevated high-sensitivity C-reactive protein: a secondary analysis from the JUPITER (justification for the use of statins in prevention-an intervention trial evaluating rosuvastatin) trial. J Am Coll Cardio/ 2010, 55(12):1266-1273.

34. Tonelli M, Moye L, Sacks FM, Kiberd B, Curhan G: Pravastatin for secondary prevention of cardiovascular events in persons with mild chronic renal insufficiency. Ann Intern Med 2003, 138(2):98-104.

35. Brouwers FP, Asselbergs FW, Hillege HL, de Boer RA, Gansevoort RT, van Veldhuisen DJ, van Gilst WH: Long-term effects of fosinopril and pravastatin on cardiovascular events in subjects with microalbuminuria: Ten years of follow-up of prevention of renal and vascular End-stage disease intervention trial (PREVEND IT). Am Heart J 2011, 161(6):1171-1178.

36. Heart Protection Study Collaborative Group: MRC/BHF Heart Protection Study of cholesterol lowering with simvastatin in 20,536 high-risk individuals: a randomised placebo-controlled trial. Lancet 2002, 360(9326):7-22.

37. Foley RN, Parfrey PS, Sarnak MJ: Epidemiology of cardiovascular disease in chronic renal disease. J Am Soc Nephrol 1998, 9(12 Suppl):S16-23.

38. Navaneethan SD, Pansini F, Perkovic V, Manno C, Pellegrini F, Johnson DW, Craig JC, Strippoli GF: HMG CoA reductase inhibitors (statins) for people with chronic kidney disease not requiring dialysis. Cochrane Database Syst Rev 2009, 2, CD007784.

39. National Kidney Foundation: K/DOQI Clinical Practice Guidelines for Anemia of Chronic Kidney Disease. Am J Kidney Dis 2000, 37(1 suppl):182-238.

40. Liu Y, Coresh J, Eustace JA, Longenecker JC, Jaar B, Fink NE, Tracy RP, Powe NR, Klag MJ: Association between cholesterol level and mortality in dialysis patients: role of inflammation and malnutrition. JAMA 2004, 291(4):451-459.

41. Bonetti $P O$, Lerman LO, Napoli C, Lerman A: Statin effects beyond lipid lowering-are they clinically relevant? Eur Heart J 2003, 24(3):225-24.

\section{doi:10.1186/1471-2261-14-19}

Cite this article as: Zhang et al:: Effect of statins on cardiovascular events in patients with mild to moderate chronic kidney disease: a systematic review and meta-analysis of randomized clinical trials. BMC Cardiovascular Disorders 2014 14:19. 